\title{
ESSENTIAL SPECTRUM OF THE LINEARIZED 2D EULER EQUATION AND LYAPUNOV-OSELEDETS EXPONENTS
}

\author{
ROMAN SHVYDKOY AND YURI LATUSHKIN
}

\begin{abstract}
The linear stability of a steady state solution of 2D Euler equations of an ideal fluid is being studied. We give an explicit geometric construction of approximate eigenfunctions for the linearized Euler operator $L$ in vorticity form acting on Sobolev spaces on two dimensional torus. We show that each nonzero Lyapunov-Oseledets exponent for the flow induced by the steady state contributes a vertical line to the essential spectrum of $L$. Also, we compute the spectral and growth bounds for the group generated by $L$ via the maximal Lyapunov-Oseledets exponent. When the flow has arbitrarily long orbits, we show that the essential spectrum of $L$ on $L_{2}$ is the imaginary axis.
\end{abstract}

\section{INTRODUCTION}

Let $u=u(x)$ be a $C^{\infty}$-steady state solution of the Euler equations governing the motion of an inviscid ideal fluid:

$$
\partial_{t} u+\langle u, \nabla\rangle u+\nabla P=0, \quad \operatorname{div} u=0 .
$$

Here $u$ is the velocity, $P$ is the pressure, and $\langle\cdot, \cdot\rangle$ denotes the scalar product. The study of the spectrum of the linearized Euler operator $L$ obtained by linearization of the Euler equations about the steady state and the spectrum of the group $\left\{e^{t L}\right\}$ has a long history, see [C, DH, DR, FH, L, Y].

Recently, an important breakthrough has been made in understanding the essential spectrum of $L$ and $e^{t L}$, see $[\mathrm{FV}, \mathrm{FV} 2, \mathrm{FSV}, \mathrm{FSV} 2$, LH1, LH2, V, VF] and the bibliography therein. In particular, using asymptotic expansions for integral Fourier operators, the boundary of the essential spectrum of $e^{t L}$ (in dimensions two and three) was related

Date: October 24, 2018.

The authors wish to thank S. Friedlander and M. Vishik for many stimulating discussions. Second author was partially supported by the Twinning Program of the National Academy of Sciences and National Science Foundation, and by the Research Council and Research Board of the University of Missouri. 
to the maximal Lyapunov exponent of a so-called bicharacteristic amplitude system, see [V, VF, S2] and also (8) below. These equations are obtained by substituting a high frequency oscillating anzats into Euler equations. As a result, it was discovered in [FV, FV2] that the presence of positive Lyapunov exponents for the flow induced by the steady state leads to the linear hydrodynamic instability of the fluid. Later, using the bicharacteristic amplitude system, results from [V], and a construction of highly oscillating approximate eigenfunctions for $L$, the boundaries of the essential spectra of $L$ and $e^{t L}$ for velocity in $L_{2}$ were related in [LV]. Note that in dimension two the maximal Lyapunov exponent of the bicharacteristic amplitude system is equal to the maximal Lyapunov exponent of the flow induced by the steady state.

In the current paper (for dimension two) we propose an approach that does not require either the use of the bicharacteristic amplitude system or the high frequency asymptotic expansions, and give an explicit construction of approximate eigenfunctions for the linearized Euler operator on all Sobolev spaces working directly with the flow induced by the steady state. This construction is related to the one used in [LV]. Also, we take a look inside the essential spectrum and show that each nonzero Lyapunov-Oseledets exponent of the flow contributes a whole vertical line to the spectrum. This also gives a formula for the boundaries of the essential spectra of $L$ and $e^{t L}$ in terms of the Lyapunov exponents for the flow generated by $u$.

In the subsequent work [SL], using the results of the current paper, we proved that the essential spectrum of $L$ in dimension two fills a solid vertical strip. However, the formulas for the approximate eigenfunctions presented here allow one to prove that the Lyapunov-Oseledets exponents generate vertical lines in the essential spectrum of the linearized surface quasi-geostrophic equation [S].

We study the linearized Euler operator $L$ in vorticity form,

$$
L w=-\langle u, \nabla\rangle w-\left\langle\operatorname{curl}^{-1} w, \nabla\right\rangle \operatorname{curl} u,
$$

on the Sobolev spaces $H_{m}^{0}=H_{m}^{0}\left(\mathbb{T}^{2} ; \mathbb{C}\right), m \in \mathbb{Z}$, of scalar functions $w$ having zero means $\int w d x=0$ on the 2 -torus $\mathbb{T}^{2}=\mathbb{R}^{2} / 2 \pi \mathbb{Z}^{2}$. We set $H_{0}^{0}=L_{2}^{0}\left(\mathbb{T}^{2} ; \mathbb{C}\right)$. See Section 2 for an explanation how the operator $L$ in vorticity form is related to the linearization of the Euler equation (1) for velocity.

Our main observation is that in the representation $L=-A+K$, where $K w=-\left\langle\operatorname{curl}^{-1} w, \nabla\right\rangle \operatorname{curl} u$ is a compact operator, the operator $A, A w=\langle u, \nabla\rangle w$, generates a so-called evolution, or Mather semigroup $e^{t A} w=w \circ \varphi_{t}$. Here and below $\varphi_{t}: x_{0} \mapsto x\left(t ; x_{0}\right)$ is the flow on $\mathbb{T}^{2}$ 
induced by the steady state velocity field, that is, by the solutions of the equation $\partial_{t} x(t)=u(x(t))$. Note that $A^{*}=-A$ with respect to the $L_{2}$-paring.

The spectral theory of the evolution semigroups is fairly well understood, see [CL] and the bibliography therein. In particular, there are several known ways to construct approximate eigenfunctions for the operators $A$ and $e^{t A}$. We stress that the construction of approximate eigenfunctions proposed in the current paper is much easier than those in $[\mathrm{CL}]$.

\section{Notation AND Preliminaries}

For an operator $B$ on a Hilbert space $\mathcal{H}$ we denote by $\sigma(B)=$ $\sigma(B ; \mathcal{H})$ its spectrum, that is, the set of all $z \in \mathbb{C}$ such that $B-z I$ does not have a bounded inverse. We denote by $\sigma_{\text {ess }}(B)=\sigma_{\text {ess }}(B ; \mathcal{H})$ the essential (Weyl) spectrum, that is, the set of all $z \in \sigma(B)$ such that $z$ is not an isolated eigenvalue of finite algebraic multiplicity (see, e.g., [EE] for a detailed discussion of various notions of the essential spectrum). We let $\operatorname{rsp}(B)$ and $\operatorname{rsp} \operatorname{ess}(B)$ denote the spectral radius and essential spectral radius of a bounded operator $B$. Recall, that Nussbaum's formula for essential spectral radius reads $[\mathrm{N}]$ :

$$
\operatorname{rsp} \operatorname{ess}(B)=\lim _{n \rightarrow \infty}\left(\inf _{K}\left\|B^{n}+K\right\|\right)^{1 / n}
$$

where the inf is taken over the set of compact operators on $\mathcal{H}$.

If $B$ is a generator of a strongly continuous semigroup $\left\{e^{t B}\right\}_{t \geq 0}$ on $\mathcal{H}$, then

$$
\omega(B)=t^{-1} \log \operatorname{rsp}\left(e^{t B}\right)=\lim _{\tau \rightarrow \infty} \tau^{-1} \log \left\|e^{\tau B}\right\|
$$

and $\omega_{\text {ess }}(B)=t^{-1} \log \operatorname{rsp} \operatorname{ess}\left(e^{t B}\right), t \neq 0$, denote the growth bound and the essential growth bound of the semigroup. Let

$$
s(B)=\sup \{\operatorname{Re} z: z \in \sigma(B)\}
$$

denote the spectral bound. Remark that $\omega(B) \geq s(B)$ for all strongly continuous semigroups on $\mathcal{H}$. However, the inverse inequality is, generally, false, see, e.g., [EN] for a discussion and further references on this topic.

We say that $z \in \mathbb{C}$ is an approximate eigenvalue and a sequence $\left\{g_{n}\right\}_{n=1}^{\infty}$ is an approximate eigenfunction for $B$ if $\left\|g_{n}\right\|=1, g_{n} \in \operatorname{Dom} B$, and $\lim _{n \rightarrow \infty}\left\|(B-z) g_{n}\right\|=0$. We say that an approximate eigenfunction is weakly null if, in addition, w- $\lim _{n \rightarrow \infty} g_{n}=0$ for the weak limit.

For $m \in \mathbb{N}$ we let $\mathcal{B}_{m}$ denote the set of $m$-linear operators $B$ with $\|B\|_{\mathcal{B}_{m}}=\sup \left\{\left|B\left(v_{1}, \ldots, v_{m}\right)\right|:\left|v_{1}\right|=\ldots=\left|v_{m}\right|=1\right\}$. We write $c$ for a generic constant, $a \lesssim b$ if $a \leq c b$, and denote by $\mathbf{1}_{[a, b]}$ the 
characteristic function of the interval $[a, b]$. We use symbol "T" to denote transposition.

Let $\mathcal{M}=\mathcal{M}\left(\left\{\varphi_{t}\right\}\right)$ denote the set of $\varphi_{t}$-invariant Borel probability measures on $\mathbb{T}^{2}$, and $\Sigma$ denote the set of all Lyapunov-Oseledets exponents $\lambda=\lambda(\nu)$ for the differential $\left\{D \varphi_{t}\right\}_{t \in \mathbb{R}}$ given for each $\nu \in \mathcal{M}$ by the Oseledets' Multiplicative Ergodic Theorem [O], see Section 4 for more details. Denote $\Lambda=\sup \{\lambda(\nu): \nu \in \mathcal{M}\}$. Recall, see [CL, Thm.8.15], that

$$
\Lambda=\lim _{t \rightarrow \infty} t^{-1} \log \max _{x \in \mathbb{T}^{2}}\left\|D \varphi_{t}(x)\right\| .
$$

Going back to Euler equation (1), consider its linearization about the steady-state $u$. The corresponding linear operator $L_{\mathrm{vel}}$ acts on (divergence free, velocity) vector fields by the rule

$$
L_{\mathrm{vel}} v=-\langle u, \nabla\rangle v-\langle v, \nabla\rangle u-\nabla P .
$$

The operator $L_{\mathrm{vel}}$ with the maximal domain $\left\{v \in H_{m}^{s}: L_{\mathrm{vel}} v \in H_{m}^{s}\right\}$ will be considered on the space $H_{m}^{s}=H_{m}^{s}\left(\mathbb{T}^{2} ; \mathbb{C}^{2}\right)$ of divergence free vector fields from the Sobolev space $H_{m}\left(\mathbb{T}^{2} ; \mathbb{C}^{2}\right), m \in \mathbb{Z}$. Note that because we are in the two dimensional situation, vorticity $w=\operatorname{curl} v$ is a scalar function. Throughout, by $\operatorname{curl} v$ we mean the scalar curl of a two dimensional vector field $v=\left(v_{1}, v_{2}\right)^{\top}$, that this, $w=\operatorname{curl} v=$ $-\partial_{2} v_{1}+\partial_{1} v_{2}$. If $w$ is a scalar function on $\mathbb{T}^{2}$ having zero mean, then we denote by $v=\operatorname{curl}^{-1} w$ the unique solution of the system $\operatorname{curl} v=w$, $\operatorname{div} v=0$ on $\mathbb{T}^{2}$.

Passing to the Fourier transform $w(x)=\sum_{\mathbf{k} \in \mathbb{Z}^{2}} w_{\mathbf{k}} e^{i \mathbf{k} \cdot x}, w_{0}=0$, $\mathbf{k}=\left(k_{1}, k_{2}\right)^{\top} \in \mathbb{Z}^{2}, x \in \mathbb{T}^{2}$, we have:

$$
v(x)=\operatorname{curl}^{-1} w(x)=\sum_{\mathbf{k} \in \mathbb{Z}^{2} \backslash\{0\}} \frac{\left(-k_{2}, k_{1}\right)^{\top}}{\|\mathbf{k}\|^{2}} w_{\mathbf{k}} e^{i \mathbf{k} \cdot x} .
$$

Therefore, the operator curl $: H_{m}^{s}\left(\mathbb{T}^{2} ; \mathbb{C}^{2}\right) \rightarrow H_{m-1}^{0}\left(\mathbb{T}^{2} ; \mathbb{C}\right), m \in \mathbb{Z}$, is an isomorphism.

For the operator $L_{\mathrm{vel}}$ defined in (5), and the operator $L$ defined in (2), we note the identity

$$
\operatorname{curl}^{-1} L \operatorname{curl} v=L_{\mathrm{vel}} v .
$$

Indeed, since both $u$ and $v$ in (5) are divergence free, by standard vector identities we infer:

$$
\begin{aligned}
\operatorname{curl} L_{\mathrm{vel}} v & =\operatorname{curl}(-\langle u, \nabla\rangle v-\langle v, \nabla\rangle u-\nabla P) \\
& =-\langle u, \nabla\rangle \operatorname{curl} v-\langle v, \nabla\rangle \operatorname{curl} u=L \operatorname{curl} v .
\end{aligned}
$$


Remark 1. Since the operators $L: H_{m}^{0}\left(\mathbb{T}^{2} ; \mathbb{C}\right) \rightarrow H_{m}^{0}\left(\mathbb{T}^{2} ; \mathbb{C}\right)$ and $L_{\text {vel }}: H_{m-1}^{s}\left(\mathbb{T}^{2} ; \mathbb{C}^{2}\right) \rightarrow H_{m-1}^{s}\left(\mathbb{T}^{2} ; \mathbb{C}^{2}\right)$ are similar by $(7)$, we conclude that their spectra in respective spaces are equal.

Finally, since the symbol $\left\{\left(-k_{2}, k_{1}\right)^{\top} /\|\mathbf{k}\|^{2}\right\}_{\mathbf{k} \in \mathbb{Z}^{2}}$ in (6) tends to zero as $\|\mathbf{k}\| \rightarrow \infty$, we remark that the operator $K: H_{m}^{0} \rightarrow H_{m}^{0}$, acting on the space of $2 \pi$-periodic functions by the rule $K w=-\left\langle\operatorname{curl}^{-1} w, \nabla\right\rangle$ curl $u$, is compact for all $m \in \mathbb{Z}$.

\section{Main Results}

Let $p(x)=\inf \left\{t>0: \varphi_{t} x=x\right\}$ denote the prime period of $x \in \mathbb{T}^{2}$. We set $p(x)=\infty$ if the point $x$ is nonperiodic. We say that the flow $\left\{\varphi_{t}\right\}_{t \in \mathbb{R}}$ has arbitrarily long trajectories if for each $N \in \mathbb{N}$ there is an $x \in \mathbb{T}^{2}$ such that $p(x) \geq N$.

Theorem 2. If $m \in \mathbb{Z} \backslash\{0\}$ then $m \Sigma \backslash\{0\}+i \mathbb{R} \subset \sigma_{\text {ess }}\left(L ; H_{m}^{0}\right)$.

Theorem 3. If $\left\{\varphi_{t}\right\}$ has arbitrarily long trajectories, then
(a) $\quad \sigma_{\mathrm{ess}}\left(L ; L_{2}^{0}\right)=i \mathbb{R}$ and
(b) $\quad i \mathbb{R} \subset \sigma_{\text {ess }}\left(L ; H_{m}^{0}\right), \quad m \in \mathbb{Z}$.

These theorems also hold if $\sigma_{\text {ess }}(L)$ is replaced by $\sigma(A)$.

Note that the assumption on $\left\{\varphi_{t}\right\}$ in Theorem 3 is essential. Indeed, if $u=(1,0)^{\top}$ then $\sigma\left(L ; L_{2}^{0}\right)=2 \pi i \mathbb{Z}$. The assumption in Theorem 3 holds for many flows on $\mathbb{T}^{2}$ as shown, e. g., in the following proposition proved in Appendix.

Proposition 4. If $u$ is not identically zero and has at least two distinct stagnation points, then $\left\{\varphi_{t}\right\}$ has arbitrarily long orbits.

Theorems 2-3 and the spectral inclusion $\exp (t \sigma(L)) \subset \sigma\left(e^{t L}\right)$, see [EN, Thm.IV3.6], show that each nonzero $\lambda \in \Sigma$ generates a circle in $\sigma_{\text {ess }}\left(e^{t L}\right)$. The bounds of $\sigma_{\text {ess }}\left(e^{t L}\right)$ are given in the next result.

Theorem 5. If $m \in \mathbb{Z}$ then $\omega_{\mathrm{ess}}(L)=\omega_{\mathrm{ess}}(-L)=|m| \Lambda$ on $H_{m}^{0}$.

The proofs of these results are given in the next section. Passing to the dual space $H_{-m}^{0}$, if necessary, we can assume that $m \geq 0$. Our plan for the proof of Theorems 2 and 3 is to construct a sequence of approximate eigenfunctions for the operator $A$, whose supports are stretched along a suitably chosen streamline and tend to zero in measure. That is, for Theorem 2, we will construct for each $\lambda \in \Sigma \backslash\{0\}$ and $\xi \in \mathbb{R}$ a weakly null approximate eigenfunction $\left\{g_{n}\right\} \subset H_{m}^{0}$ for the approximate eigenvalue $\lambda+i \xi$ for $A$. Then we will use the compactness of $K$ to extract a subsequence on which $\left\|K g_{j}\right\| \rightarrow 0$. Since $L=-A+K$, this way we will produce an approximative eigenfunction for $L$. The proof 
of Theorem 5 follows from the equality $\omega(A)=\omega_{\text {ess }}(A)=m \Lambda$ proved in Section 4, compactness of $K$, and Nussbaum's formula (3) for the essential spectral radius that implies $\omega_{\text {ess }}(L)=\omega_{\text {ess }}(-A)$.

Corollary 6. If $m \in \mathbb{Z}$, then $s(L)=\omega(L)$ on $H_{m}^{0}$.

This holds since $\omega(L)=\max \left\{s(L), \omega_{\text {ess }}(L)\right\}$ by [EN, Cor.IV.2.11], and $s(L) \geq|m| \Lambda=\omega_{\text {ess }}(L)$ by Theorem 2, Theorem 5, and identity $L(\operatorname{curl} u)=0$ (for $m=-1$ see [LV] for a different proof).

Let $L_{\mathrm{vel}} v=-\langle u, \nabla\rangle v-\langle v, \nabla\rangle u-\nabla P$ be the linearized Euler operator (5) in velocity form acting on the space $H_{m}^{s}=H_{m}^{s}\left(\mathbb{T}^{2} ; \mathbb{C}^{2}\right)$ of divergence free vector fields $v$. We have $\sigma\left(L ; H_{m}^{0}\right)=\sigma\left(L_{\mathrm{vel}} ; H_{m+1}^{s}\right), m \in \mathbb{Z}$, by Remark 1. Thus, all results above can be reformulated for $L_{\mathrm{vel}}$. In particular, $\omega_{\mathrm{ess}}\left(L_{\mathrm{vel}} ; H_{m}^{s}\right)=|m-1| \Lambda$ and $\omega\left(L_{\mathrm{vel}} ; H_{m}^{s}\right)=s\left(L_{\mathrm{vel}} ; H_{m}^{s}\right)$, $m \in \mathbb{Z}$. To relate these results to the work in $[\mathrm{V}, \mathrm{VF}]$, let

$$
\begin{gathered}
\mu=\lim _{t \rightarrow+\infty} t^{-1} \log \max \left\{\left|b\left(t ; x_{0}, \xi_{0}, b_{0}\right)\right|:\left(x_{0}, b_{0}, \xi_{0}\right) \in \mathbb{T}^{2} \times\left(\mathbb{R}^{2}\right)^{*} \times \mathbb{R}^{2},\right. \\
\left.\left|b_{0}\right|=\left|\xi_{0}\right|=1 \quad \text { and } \quad \xi_{0} \perp b_{0}\right\}
\end{gathered}
$$

denote the maximal Lyapunov exponent for the $b$-equation of the following bicharacteristic amplitude system:

$$
\begin{aligned}
\partial_{t} x & =u(x), \quad \partial_{t} \xi=-(D u)^{\top} \xi, \\
\partial_{t} b & =-(D u) b+2\langle(D u) b, \xi\rangle \xi|\xi|^{-2} .
\end{aligned}
$$

Here and below $D u=\partial u / \partial x$ is the Jacobi matrix, and we write $D u^{-\top}=\left((D u)^{\top}\right)^{-1}$. Note that $|b(t)||\xi(t)|$ is a first integral for (8), see [FV2]. The Multiplicative Ergodic Theorem, therefore, implies that $\Sigma$ coincides with the Lyapunov-Oseledets spectrum of the cocycle generated by $b$-equation in (8) and, in particular, $\Lambda=\mu$, cf. [FV2]. It was proved in $[\mathrm{V}, \mathrm{VF}]$ that $\omega_{\mathrm{ess}}\left(L_{\mathrm{vel}} ; L_{2}^{s}\right)=\mu$. Thus, we have a generalization of this formula for any $m \in \mathbb{Z}$. Also, note the estimate $\omega_{\text {ess }}\left(L_{\mathrm{vel}}, H_{m}^{s}\right) \geq \mu_{m}$ given in $[\mathrm{FV}]$. Here $\mu_{m}$ is the maximal Lyapunov exponent for $\left(1+|\xi|^{2}\right)^{m / 2} b(t)$. This estimate is in tune with the inequality " $\geq$ " in Theorem 5 .

Remark 7. In the subsequent paper [SL], using the results above and a modification of the construction used in the proof of Theorems 2 and 3 , we show that, in fact, $\sigma_{\mathrm{ess}}\left(L ; H_{m}^{0}\right)=\{z \in \mathbb{C}:|\operatorname{Re} z| \leq|m| \Lambda\}$.

\section{Proofs}

Recall the statement of the Multiplicative Ergodic Theorem for the cocycle $\left\{D \varphi_{t}\right\}_{t \in \mathbb{R}}$, see $[\mathrm{O}]$ : For each $\nu \in \mathcal{M}$ there exists a full $\nu$-measure subset $X_{\nu} \subset \mathbb{T}^{2}$ such that for each $x \in X_{\nu}$ and each nonzero $v \in \mathcal{T}_{x} \mathbb{T}^{2}$, 
the tangent space at $x \in \mathbb{T}^{2}$, the following forward and backward exact Lyapunov exponents exist and are equal:

$$
\lambda(x, v):=\lim _{t \rightarrow+\infty} t^{-1} \log \left|D \varphi_{t}(x) v\right|=\lim _{t \rightarrow-\infty} t^{-1} \log \left|D \varphi_{t}(x) v\right| .
$$

Since the cocycle $\left\{D \varphi_{t}\right\}$ is two dimensional, for each $x \in X_{\nu}$ and all $v \in \mathcal{T}_{x} \mathbb{T}^{2}$ there exist at most two different Lyapunov exponents that we will denote by $\lambda_{1}$ and $\lambda_{2}, \lambda_{1} \geq \lambda_{2}$. We stress that $\lambda_{1,2}=\lambda_{1,2}(\nu)$, and we denote $\Lambda=\max \left\{\lambda_{1}(\nu): \nu \in \mathcal{M}\right\}$.

Since $\operatorname{div} u=0$, we have $\operatorname{det} D \varphi_{t}(x)=1$ for all $x \in \mathbb{T}^{2}$. This implies $\lambda_{1}+\lambda_{2}=0$ for all $x \in X_{\nu}$ and $\nu \in \mathcal{M}[\mathrm{O}]$. If $y$ is a stagnation point for $u$, then $D \varphi_{t}(y)=e^{t D u(y)}$ and $\operatorname{Re} \sigma(D u(y))=\left\{\lambda_{1}, \lambda_{2}\right\}$ for the Lyapunov exponents $\lambda_{1,2}$ at $y$. Since $D u(y)$ is a matrix with real entries and zero trace, if $\lambda_{1} \neq 0$ then, in fact, $\sigma(D u(y))=\left\{-\lambda_{1}, \lambda_{1}\right\}$.

Remark 8. If $\lambda \in \Sigma \backslash\{0\}$ then there exists a stagnation point $y$ such that $\lambda$ is a Lyapunov exponent at $y$, cf. [FSV2]. To see this, fix $\nu \in \mathcal{M}$ and $x \in X_{\nu}$ such that $\lambda=\lambda(x, v)$ for some $v \in \mathcal{T}_{x} \mathbb{T}^{2} \backslash\{0\}$. Suppose that $u(x) \neq 0$. Since $\max _{x \in \mathbb{T}^{2}}|u(x)|<\infty$, the identity

$$
D \varphi_{t}(x) u(x)=u\left(\varphi_{t} x\right), \quad t \in \mathbb{R}, \quad x \in \mathbb{T}^{2},
$$

implies that the forward Lyapunov exponent for $u(x)$ is nonpositive. By the same reason the backward Lyapunov exponent for $u(x)$ is nonnegative. Thus, $\lambda(x, u(x))=0$. This implies $\lambda_{1}=\lambda_{2}=0$, in contradiction with $\lambda=\lambda(x, v) \neq 0$. Thus $x=y$, a stagnation point.

Remark 9. Assume that $\lambda \in \Sigma$ and $\lambda>0$. By Remark 8, find a hyperbolic stagnation point $y$ such that $\sigma(D u(y))=\{-\lambda, \lambda\}$. If $v \in \mathcal{T}_{y} \mathbb{T}^{2},|v|=1$, is the eigenvector for $D u(y)$ such that $D u(y)=-\lambda v$, then by the Stable Manifold Theorem there is a manifold $\mathcal{O}$ that is tangent to $v$ at $y$. In other words, if $x_{0} \rightarrow y$ such that $x_{0} \in \mathcal{O}$, then $u\left(x_{0}\right) /\left|u\left(x_{0}\right)\right| \rightarrow v$. Also, for each $t \in \mathbb{R}$, if $x_{0} \rightarrow y, x_{0} \in \mathcal{O}$, then $D \varphi_{t}\left(x_{0}\right) \rightarrow e^{t D u(y)}$. Using (9), we conclude that

$$
\lim _{\mathcal{O} \ni x_{0} \rightarrow y} u\left(\varphi_{t} x_{0}\right) /\left|u\left(x_{0}\right)\right|=e^{-\lambda t} v, \quad t \in \mathbb{R} .
$$

Proofs of Theorems 2 and 3. As explained in Section 3, it suffices to construct a weakly null approximate eigenfunction $\{g\}$ for $A$ such that all functions $g$ are localized along a streamline. For technical reasons it is more convenient to work on the plane with straightened streamlines. Therefore, we introduce the necessary volume-preserving change of variables localized around an orbit of $\left\{\varphi_{t}\right\}$. After this change of variables $A$ becomes simply the differentiation. 
Fix an $N \in \mathbb{N}$ and a point $x_{0} \in \mathbb{T}^{2}$ such that $u\left(x_{0}\right) \neq 0$ and $p\left(x_{0}\right)>$ $3 N$. For $u=\left(u_{1}, u_{2}\right)^{\top}$ denote $u^{\perp}=\left(-u_{2}, u_{1}\right)^{\top}$. Let $\left\{\psi_{\tau}\right\}$ be the local flow at $x_{0}$ such that

$$
\partial_{\tau}\left(\psi_{\tau}\left(x_{0}\right)\right)=\frac{u^{\perp} \circ \psi_{\tau}\left(x_{0}\right)}{\left|u \circ \psi_{\tau}\left(x_{0}\right)\right|^{2}} .
$$

Define a mapping $H(t, \tau)=\varphi_{t} \circ \psi_{\tau}\left(x_{0}\right)$ for $|t|<p\left(x_{0}\right) / 2$ and $|\tau|$ small enough to ensure the injectivity of $H$. So, $H$ is defined on a horizontal strip $\mathcal{S}=[-N, N] \times[-s, s]$. From the definition of $H$ we obtain:

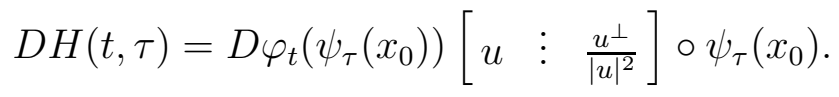

Thus, $\operatorname{det} D H=1$ and $H$ is volume-preserving. Using this, one easily computes

$$
\begin{aligned}
D H^{-\top}(t, \tau) \stackrel{\text { def }}{=} D H^{-\top} & (H(t, \tau)) \\
& =\left[D \varphi_{t}\left(\psi_{\tau}\left(x_{0}\right)\right)\right]^{-\top}\left[\begin{array}{lll}
\frac{u}{|u|^{2}} & \vdots & u^{\perp}
\end{array}\right] \circ \psi_{\tau}\left(x_{0}\right) .
\end{aligned}
$$

Given a function $F$ supported on $\mathcal{S}$ put $f=F \circ H^{-1}$. Then

$$
\nabla f(H(t, \tau))=D H^{-\top}(t, \tau) \nabla F(t, \tau) .
$$

More generally, for $D^{m} f \in \mathcal{B}_{m}$, the $m$-th differential of $f$, by the chain rule (see, e. g., [AMR, p.97]) we have:

$$
\begin{array}{r}
D^{m} f(H(t, \tau))\left(v_{1}, \ldots, v_{m}\right)=D^{m} F(t, \tau)\left(D H^{-1} v_{1}, \ldots, D H^{-1} v_{m}\right) \\
+ \text { lower order derivatives of } F
\end{array}
$$

Fix an $\alpha=\lambda+i \xi \in \mathbb{C}$. Define

$$
F(t, \tau)=e^{\alpha t} \gamma(t) \beta(\tau) \quad \text { for } \quad(t, \tau) \in[-N, N] \times[-s, s] .
$$

A direct calculation shows:

$$
A f-\left.\alpha f\right|_{H(t, \tau)}=\tilde{F}(t, \tau), \quad \text { where } \quad \tilde{F}(t, \tau) \stackrel{\text { def }}{=} e^{\alpha t} \gamma^{\prime}(t) \beta(\tau) .
$$

To make the main idea of the proof more transparent, we first consider the case $m=1$. The general case $m \geq 1$ will be considered later.

We compute:

$$
\nabla F=\left[\begin{array}{c}
\alpha F+e^{\alpha t} \gamma^{\prime}(t) \beta(\tau) \\
e^{\alpha t} \gamma(t) \beta^{\prime}(\tau)
\end{array}\right] ; \quad \nabla \tilde{F}=\left[\begin{array}{c}
\alpha \tilde{F}+e^{\alpha t} \gamma^{\prime \prime}(t) \beta(\tau) \\
e^{\alpha t} \gamma^{\prime}(t) \beta^{\prime}(\tau)
\end{array}\right]
$$

Let us choose $\gamma(t)=\left(1-|t| N^{-1}\right) \chi_{[-N, N]}$ smoothed out at $\pm N, 0$, and $\beta(\tau)=(s-|\tau|) \chi_{[-s, s]}$. Clearly, $\frac{1}{2 s}\left(\beta^{\prime}(\tau)\right)^{2}$ is an approximative kernel. 
So, if $s \rightarrow 0$, then

$$
\begin{aligned}
& \frac{1}{2 s}|\partial F / \partial t|^{2} \rightarrow 0, \quad \frac{1}{2 s}|\partial \tilde{F} / \partial t|^{2} \rightarrow 0 \text { in } L_{1}, \\
& \frac{1}{2 s}|\partial F / \partial \tau|^{2} \rightarrow e^{2 \lambda t}|\gamma(t)|^{2} \delta_{0}(\tau) \\
& \frac{1}{2 s}|\partial \tilde{F} / \partial \tau|^{2} \rightarrow e^{2 \lambda t}\left|\gamma^{\prime}(t)\right|^{2} \delta_{0}(\tau) .
\end{aligned}
$$

Here $\delta_{0}$ denotes the Dirac $\delta$-function, and the last two limits in (17) are understood in the sense of distributions. Furthermore, the measure of the support of $f=F \circ H^{-1}$ tends to zero as $s \rightarrow 0$. So, $f /\|f\|_{H_{1}}$ converges to zero weakly, and is norm bounded as $s \rightarrow 0$. Since $K$ is a compact operator, we therefore conclude that $\left\|K\left(f /\|f\|_{H_{1}}\right)\right\|_{H_{1}} \rightarrow 0$. Passing to the $(t, \tau)$-coordinates in integrals and using (11) we have, as $s \rightarrow 0$,

$$
\begin{aligned}
\|L+\alpha\|_{\bullet}^{2} & \stackrel{\text { def }}{=} \inf \left\{\|L g+\alpha g\|_{H_{1}}^{2}:\|g\|_{H_{1}}=1\right\} \\
& \lesssim\|A f-\alpha f\|_{H_{1}}^{2} /\|f\|_{H_{1}}^{2}+\left\|K\left(f /\|f\|_{H_{1}}\right)\right\|_{H_{1}}^{2}
\end{aligned}
$$

by (12) and (15),

$$
=\frac{(2 s)^{-1} \int_{\mathcal{S}}\left|D H^{-\top} \nabla \tilde{F}\right|^{2} d \tau d t}{(2 s)^{-1} \int_{\mathcal{S}}\left|D H^{-\top} \nabla F\right|^{2} d \tau d t}+\left\|K\left(f /\|f\|_{H_{1}}\right)\right\|_{H_{1}}^{2}
$$

by (11) and (17),

$$
\rightarrow \frac{\int_{\mathbb{R}}\left|\left[D \varphi_{t}\left(x_{0}\right)\right]^{-\top} u^{\perp}\left(x_{0}\right)\right|^{2} e^{2 \lambda t}\left|\gamma^{\prime}(t)\right|^{2} d t}{\int_{\mathbb{R}}\left|\left[D \varphi_{t}\left(x_{0}\right)\right]^{-\top} u^{\perp}\left(x_{0}\right)\right|^{2} e^{2 \lambda t}|\gamma(t)|^{2} d t} .
$$

Using the identity

$$
\left[D \varphi_{t}\left(x_{0}\right)\right]^{-\top} u^{\perp}\left(x_{0}\right)=u^{\perp} \circ \varphi_{t}\left(x_{0}\right)
$$

the last expression is equal to

$$
\frac{\int_{\mathbb{R}}\left|u \circ \varphi_{t}\left(x_{0}\right)\right|^{2} /\left|u\left(x_{0}\right)\right|^{2} e^{2 \lambda t}\left|\gamma^{\prime}(t)\right|^{2} d t}{\int_{\mathbb{R}}\left|u \circ \varphi_{t}\left(x_{0}\right)\right|^{2} /\left|u\left(x_{0}\right)\right|^{2} e^{2 \lambda t}|\gamma(t)|^{2} d t} .
$$

Fix a nonzero Lyapunov exponent $\lambda \in \Sigma$ and any $\xi \in \mathbb{R}$. By Remark 8 there is a hyperbolic stagnation point $y$ such that $\lambda$ is a Lyapunov exponent at $y$. Pick $v \in \mathcal{T}_{y} \mathbb{T}^{2},|v|=1$, such that $D \varphi_{t}(y) v=e^{-\lambda t} v$. Assume for the moment that $\lambda>0$. Using Remark 9, pick a nonperiodic point $x_{0}$ that belongs to the manifold $\mathcal{O}$ tangent to $v$ at $y$. By (10), we have that $u \circ \varphi_{t}\left(x_{0}\right) /\left|u\left(x_{0}\right)\right|$ converges to $e^{-\lambda t} v$ as $x_{0} \rightarrow y$ along this 
manifold. Use the calculation above with $\alpha=\lambda+i \xi$. Passing to the limit as $x_{0} \rightarrow y, x_{0} \in \mathcal{O}$, in (19), we obtain

$$
\|L+\alpha\|_{\bullet}^{2} \leq \frac{\int_{\mathbb{R}}\left|\gamma^{\prime}(t)\right|^{2} d t}{\int_{\mathbb{R}}|\gamma(t)|^{2} d t}
$$

for arbitrary $N>0$. Observe that the quantity on the right hand side tends to zero as $N \rightarrow \infty$. The argument for $\lambda<0$ is similar.

Finally, to make $f$ mean-zero define another function $\bar{f}$ in the same way around same streamline and disjoint from $f$. Varying its support we can obtain the equality $\int_{\mathbb{T}^{2}} f d x=\int_{\mathbb{T}^{2}} \bar{f} d x$. Then $f-\bar{f}$ form the required sequence of approximate eigenfunctions. In the sequel, we refer to this procedure as symmetrization.

This finishes the proof of Theorem 2 for $m=1$.

To prove part (a) in Theorem 3 , set $\alpha=i \xi, \beta=\mathbf{1}_{[-s, s]}$ and keep $\gamma$ the same as before. Then, as $s \rightarrow 0$, we obtain:

$$
\begin{aligned}
\|L+\alpha\|_{\bullet}^{2} & \lesssim\|A f-\alpha f\|_{L_{2}}^{2} /\|f\|_{L_{2}}^{2}+\left\|K\left(f /\|f\|_{L_{2}}\right)\right\|_{L_{2}}^{2} \\
& =\frac{(2 s)^{-1} \int_{\mathcal{S}}|\tilde{F}|^{2} d \tau d t}{(2 s)^{-1} \int_{\mathcal{S}}|F|^{2} d \tau d t}+\left\|K\left(f /\|f\|_{L_{2}}\right)\right\|_{L_{2}}^{2} \rightarrow \frac{\int_{\mathbb{R}}\left|\gamma^{\prime}(t)\right|^{2} d t}{\int_{\mathbb{R}}|\gamma(t)|^{2} d t} .
\end{aligned}
$$

Under the assumption on $\left\{\varphi_{t}\right\}, N$ can be taken arbitrarily large. Symmetrization is carried out similarly. So, we have proved that $i \mathbb{R} \subset$ $\sigma_{\text {ess }}(L) \cap \sigma(A)$. On the other hand, since $A$ is antisymmetric on $L_{2}^{0}$, $\sigma(A) \subset i \mathbb{R}$. Applying a version of Weyl's Theorem as in [RS, Corollary XIII.4.2], we have $\sigma_{\text {ess }}(L)=\sigma_{\text {ess }}(A)=i \mathbb{R}$.

We continue the proof of Theorem 2 for $m \geq 1$. Define $F$ as in (14), with the same $\gamma, \alpha=m \lambda+i \xi$ and the cut-off function $\beta$ chosen such that if $s \rightarrow 0$ then the following three conditions are satisfied (see Appendix for a construction of $\beta$ ):

a) for all $k=0,1, \ldots, m-1$ the derivatives $\beta^{(k)}(\tau)$ tend to zero uniformly for $\tau \in[-s, s]$;

b) $\left|\beta^{(m)}\right|_{[-s c, s c]} \mid>1 / 2$ for some fixed $c>0$;

c) the norms $\left\|\beta^{(m)}\right\|_{\infty}$ are uniformly bounded.

This implies that, whenever $k<m$,

$$
\frac{1}{s}\left|\frac{\partial^{k+l} F}{\partial^{l} t \partial^{k} \tau}\right|^{2} \rightarrow 0, \quad \frac{1}{s}\left|\frac{\partial^{k+l} \tilde{F}}{\partial^{l} t \partial^{k} \tau}\right|^{2} \rightarrow 0 .
$$

in $L_{1}$, as $s \rightarrow 0$. 
On the other hand, for some sequence $s_{j} \rightarrow 0$,

$$
\begin{aligned}
& \frac{1}{s_{j}}\left|\frac{\partial^{m} F}{\partial^{m} \tau}\right|^{2} \rightarrow c e^{2 \lambda m t}|\gamma(t)|^{2} \delta_{0}(t), \\
& \frac{1}{s_{j}}\left|\frac{\partial^{m} \tilde{F}}{\partial^{m} \tau}\right|^{2} \rightarrow c e^{2 \lambda m t}\left|\gamma^{\prime}(t)\right|^{2} \delta_{0}(t),
\end{aligned}
$$

and $\left\|K\left(f /\|f\|_{H_{m}}\right)\right\|_{H_{m}} \rightarrow 0$, where $f \circ H=F$.

As before we want to estimate the quantity $\|L+\alpha\|_{\text {: }}^{2}$ and prove that it is zero. To this end, we notice (cf. (13) and (18)) that

$$
\begin{aligned}
&\|L+\alpha\|_{\bullet}^{2} \lesssim \frac{s_{j}^{-1} \int_{\mathcal{S}}\left\|D^{m} \tilde{F}\left(D H^{-1} \cdot, \ldots, D H^{-1} \cdot\right)\right\|_{\mathcal{B}_{m}}^{2}+\| \text { lower } D \tilde{F} \|^{2} d \tau d t}{s_{j}^{-1} \int_{\mathcal{S}}\left\|D^{m} F\left(D H^{-1} \cdot, \ldots, D H^{-1} \cdot\right)\right\|_{\mathcal{B}_{m}}^{2}-\| \text { lower } D F \|^{2} d \tau d t} \\
&(22) \quad+\left\|K\left(f /\|f\|_{H_{m}}\right)\right\|_{H_{m}}^{2} .
\end{aligned}
$$

Our observations in (20) and (21) indicate that the only non-vanishing (in the limit as $s_{j} \rightarrow 0$ ) term under the integrals is the one containing $\partial^{m} F / \partial^{m} \tau$. More precisely, denoting $w_{j}=\left\langle D H^{-1} v_{j}, \mathbf{e}_{2}\right\rangle$, where $\mathbf{e}_{2}=$ $(0,1)^{\top}$, we can express this term as the product

$$
\frac{\partial^{m} F}{\partial^{m} \tau} \cdot w_{1} \cdot \ldots \cdot w_{m}
$$

Using formula (11) and the identity

$$
\left[D \varphi_{t}\left(\psi_{\tau}\left(x_{0}\right)\right)\right]^{-\top} u^{\perp}\left(\psi_{\tau}\left(x_{0}\right)\right)=u^{\perp}(H(t, \tau)),
$$

we also see that

$$
\begin{aligned}
w_{j} & =\left\langle v_{j}, D H^{-\top} \mathbf{e}_{2}\right\rangle \\
& =\left\langle v_{j},\left[D \varphi_{t}\left(\psi_{\tau}\left(x_{0}\right)\right)\right]^{-\top} u^{\perp}\left(\psi_{\tau}\left(x_{0}\right)\right)\right\rangle \\
& =\left\langle v_{j}, u^{\perp} \circ H\right\rangle .
\end{aligned}
$$

In particular,

$$
\sup _{\left|v_{j}\right|=1}\left|\frac{\partial^{m} F}{\partial^{m} \tau} \cdot w_{1} \cdot \ldots \cdot w_{m}\right| \leq\left|\frac{\partial^{m} F}{\partial^{m} \tau}\right| \cdot\left|u^{\perp} \circ H\right|^{m} .
$$

Thus, passing to the limit as $j \rightarrow \infty$ in (22), we obtain

$$
\|L+\alpha\|_{\bullet}^{2} \lesssim \frac{\int_{\mathbb{R}}\left|u^{\perp} \circ \varphi_{t}\left(x_{0}\right)\right|^{2 m} e^{2 m \lambda t}\left|\gamma^{\prime}(t)\right|^{2} d t}{\int_{\mathbb{R}}\left|u^{\perp} \circ \varphi_{t}\left(x_{0}\right)\right|^{2 m} e^{2 m \lambda t}|\gamma(t)|^{2} d t} .
$$

The rest of the proof goes as in the case $m=1$. 
To show (b) in Theorem 3, assume for the moment that $\left\{\varphi_{t}\right\}$ has arbitrary long periodic orbits. Take a large $N$ and find an orbit $\mathcal{O}$ with a finite period greater than $2 N$. Pick a point $x_{0} \in \mathcal{O}$ such that

$$
\left|u\left(x_{0}\right)\right|=\max _{x \in \mathcal{O}}|u(x)|=c .
$$

Then for all $|t| \leq 1$ we have $\left|u \circ \varphi_{t}\left(x_{0}\right)\right| \geq\left\|D \varphi_{-t}\left(x_{0}\right)\right\|^{-1} c \geq M c$, where $M$ depends only on $\left\{\varphi_{t}\right\}$. Continuing from (23) with arbitrary $\alpha \in i \mathbb{R}$ we obtain

$$
\|L+\alpha\|_{\bullet}^{2} \lesssim N^{-1} c^{2 m} /\left(M^{2 m} c^{2 m}\right),
$$

which gives the desired result.

Suppose now that for some $N>0$ if $p(x)>N$ then $p(x)=\infty$ for the prime period $p(\cdot)$. Consider the set $S=p^{-1}((0, \infty))$. The set $S$ is open and $p(x) \leq N$ for all $x \in \bar{S}$. Since $\left\{\varphi_{t}\right\}$ has arbitrarily long orbits, $S \neq \mathbb{T}^{2}$. So, if $S \neq \emptyset$, then $\partial S \backslash S \neq \emptyset$. This, however, leads to a contradiction, since if $x \in \partial S \backslash S$, then on one hand $p(x) \leq N$, but on the other hand $p(x)=\infty$. We conclude that $S$ is empty and, as a consequence, $p(x)=\infty$ for all $x \in \mathbb{T}^{2}$. In particular, $u(x) \neq 0$ for all $x \in \mathbb{T}^{2}$. This allows us to bound (23) with $\lambda=0$ by the expression

$$
c^{2 m} \frac{\int_{\mathbb{R}}\left|\gamma^{\prime}(t)\right|^{2} d t}{\int_{\mathbb{R}}|\gamma(t)|^{2} d t}, \text { where } c=\frac{\max |u(x)|}{\min |u(x)|} .
$$

As before, we infer $\|L+\alpha\|_{\bullet}=0$.

Proof of Theorem 5. Since $L=-A+K$, and the operator $K$ is compact, Nussbaum's formula (3) for essential spectral radius implies that $\omega_{\text {ess }}(L)=\omega_{\text {ess }}(-A)$. Passing to the vector field $-u$, it is enough to prove that

$$
\omega(A)=\omega_{\mathrm{ess}}(A)=m \Lambda, \quad m=1,2, \ldots,
$$

(recall, that $A$ is skew-self-adjoint on $L_{2}^{0}$, that is $\omega(A)=\omega_{\text {ess }}(A)=0$ for $m=0$ ).

Note that $\omega_{\text {ess }}(A) \geq 0$. Indeed, this follows from (b) in Theorem 3 , provided $\left\{\varphi_{t}\right\}$ has arbitrary long orbits. Otherwise, all orbits are periodic and the periods are uniformly bounded. Excluding the trivial case $u \equiv 0$, pick a point $x_{0} \in \mathbb{T}^{2}$ such that $u\left(x_{0}\right) \neq 0$. Define a local flow $\left\{\psi_{\tau}\right\}$ as in the proof of Theorems 2-3 above. Take a smooth cutoff function $\beta$ supported in a small interval $[-s, s]$. For $x=\varphi_{t}\left(\psi_{\tau}\left(x_{0}\right)\right)$ set $f(x)=\beta(\tau)$. After the symmetrization we obtain $f \in H_{m}^{0}$ and $e^{t A} f=f$. To see that 1 is an eigenvalue of $e^{t A}$ of infinite multiplicity, we argue as follows. Take a periodic orbit. Make a small transversal cross-section and split the cross-section into infinitely many disjoint intervals. For each interval $I$ construct a function $f$ as above such 
that $e^{t A} f=f$ and the support of $f$ belongs to the orbit of $I$. Thus, $1 \in \sigma_{\text {ess }}\left(e^{t A}\right)$ as claimed.

Therefore, in the proof of the inequality $\omega_{\text {ess }}(A) \geq m \Lambda$ we may assume that $\Lambda>0$. By Theorem 2 for $A$ and the spectral inclusion $e^{t \sigma(A)} \subset \sigma\left(e^{t A}\right)$ we have that $e^{t(m \lambda+i \xi)} \subset \sigma_{\text {ess }}\left(e^{t A}\right)$ for each nonzero $\lambda \in \Sigma$ and all $\xi \in \mathbb{R}$. Thus $m \Lambda \leq \omega_{\text {ess }}(A)$. It remains to prove that $\omega(A) \leq m \Lambda$. This is implied by the following lemma, proved in Appendix.

\section{Lemma 10.}

$$
m \Lambda \geq \lim _{t \rightarrow \infty} t^{-1} \log \max _{x \in \mathbb{T}^{2}}\left\|D^{m} \varphi_{t}(x)\right\|_{\mathcal{B}_{m}}, \quad m=1,2, \ldots
$$

Indeed, by $(25)$ for each $m=1,2, \ldots$ and each $\epsilon>0$ there is a constant $c=c(\epsilon, m)$ such that

$$
\max _{x \in \mathbb{T}^{2}}\left\|D^{m}\left(\varphi_{k}\right)(x)\right\|_{\mathcal{B}_{m}} \leq c e^{m k(\Lambda+\epsilon)} \text { for } k=1,2, \ldots
$$

It suffices to prove that $\left\|f \circ \varphi_{k}\right\|_{H_{m}} \leq c e^{m(\Lambda+\epsilon) k}\|f\|_{H_{m}}$ for all $k \in \mathbb{N}$. Note that

$$
\left\|f \circ \varphi_{k}\right\|_{H_{m}}^{2}=\int_{\mathbb{T}} \max _{0 \leq n \leq m}\left\|D^{n}\left(f \circ \varphi_{k}\right)(x)\right\|_{\mathcal{B}_{n}}^{2} d x
$$

and apply to $f \circ \varphi_{k}$ the chain rule in [AMR, p. 97]. Using (26) for $m=j_{q}$ we have the desired result:

$$
\begin{aligned}
& \left\|D^{n}\left(f \circ \varphi_{k}\right)(x)\right\|_{\mathcal{B}_{n}} \\
& \quad \leq \sum_{p=1}^{n} \sum_{j_{1}+\cdots+j_{p}=n} \sum_{\{\ell\}}\left\|D^{p} f\left(\varphi_{k} x\right)\right\|_{\mathcal{B}_{p}} \prod_{q=1}^{p}\left\|D^{j_{q}}\left(\varphi_{k}\right)(x)\right\|_{\mathcal{B}_{j_{q}}} \\
& \quad \leq \sum_{p=1}^{n} \sum_{j_{1}+\cdots+j_{p}=n} \sum_{\{\ell\}}\left\|D^{p} f\left(\varphi_{k} x\right)\right\|_{\mathcal{B}_{p}} c \exp \left[(\Lambda+\epsilon)\left(j_{1}+\cdots+j_{p}\right) k\right] \\
& \quad \leq c(\epsilon, m) \max _{1 \leq n \leq m}\left\|D^{n} f\left(\varphi_{k} x\right)\right\|_{\mathcal{B}_{n}} \exp [(\Lambda+\epsilon) m k] .
\end{aligned}
$$

Here the summation $\sum_{\{e\}}$ for each $p$ is taken over $\ell_{1}<\cdots<\ell_{j_{1}}, \ldots$, $\ell_{j_{1}+\cdots+j_{p-1}+1}<\cdots<\ell_{p}$, see [AMR, p. 97].

\section{APPENDIXES}

1. The construction of $\beta$. Select $\phi \in C_{0}^{\infty}[-1,1]$ with $\left.\phi\right|_{[-1 / 2,1 / 2]} \equiv 1$. Let $C=\|\phi\|_{C^{m}}$. We define $\beta_{1}(\tau)=\phi(\tau / s)$ and $\beta_{2}(\tau)=\tau^{m} / m$ !, and set $\beta=\beta_{1} \beta_{2}$. Since $\left\|\beta_{1}^{(l)}\right\|_{\infty} \leq C / s^{l}$, we have $\beta^{(k)}=\sum_{l=0}^{k} C_{k}^{l} \beta_{1}^{(l)} \beta_{2}^{(k-l)}$ 
and

$$
\left|\beta^{(k)}(\tau)\right| \leq \sum_{l=0}^{k} C_{k}^{l} \frac{1}{s^{l}} \frac{|\tau|^{m-k+l}}{(m-k+l) !} \lesssim|\tau|^{m-k} .
$$

Thus, conditions a) and c) are fulfilled. Notice also that

$$
\left|\beta^{(m)}(\tau)\right| \geq\left|\beta_{1}(\tau)\right|-C \sum_{l=1}^{m} C_{m}^{l}\left(|\tau| s^{-1}\right)^{l} / l ! .
$$

So, if $0<c<1 / 2$ is such that $C \sum_{l=1}^{m} C_{m}^{l} c^{l} / l !<1 / 2$, then $\left|\beta^{m}\right|_{[-s c, s c]} \mid>$ $1 / 2$, and $b$ ) is proved.

2. Proof of Proposition 4. Let us assume the contrary. First, we rule out one simple case: there is an unstable stagnation point $x_{0}$ in the sense of Lyapunov, namely: There exists a neighborhood $U$ of $x_{0}$ such that for some sequence $x_{n} \rightarrow x_{0}$ and $t_{n} \in \mathbb{R}$ we have $\varphi_{t_{n}}\left(x_{n}\right) \notin U$. By our assumption the sequence $\left\{t_{n}\right\}$ is bounded. For a limit point $t$ then $\varphi_{t_{n}}\left(x_{n}\right) \rightarrow \varphi_{t}\left(x_{0}\right)=x_{0} \notin U$, which is a contradiction.

Fix a point $x_{0} \in \mathbb{T}^{2}$ such that $u\left(x_{0}\right) \neq 0$. Let $U$ denote the open connected component in the set $\{x: u(x) \neq 0\}$ containing $x_{0}$. Note that $U$ is linearly connected. Take a continuous path $\gamma:[0,1] \rightarrow \mathbb{T}^{2}$ such that $\gamma((0,1)) \subset U$, and $y_{0}=\gamma(0)$ and $y_{1}=\gamma(1)$ belong to the boundary $\partial U, y_{0} \neq y_{1}$. Choose neighborhoods $U_{0}$ and $U_{1}$ of $y_{0}$ and $y_{1}$, respectively, such that $\gamma([1 / 3,2 / 3]) \subset \mathbb{T}^{2} \backslash\left(U_{0} \cup U_{1}\right)$. By our assumption, $y_{0}$ and $y_{1}$ are Lyapunov stable. This implies that there are two orbits $\mathcal{O}_{0} \subset U_{0}$ and $\mathcal{O}_{1} \subset U_{1}$ intersecting $\gamma$. Find the smallest $s_{0}$ (largest $\left.s_{1}\right)$ in $[0,1]$, for which $\gamma\left(s_{0}\right) \in \mathcal{O}_{0}\left(\gamma\left(s_{1}\right) \in \mathcal{O}_{1}\right)$. Then $s_{0}<s_{1}$ and $\gamma\left(\left(s_{0}, s_{1}\right)\right)$ lies in the exteriors of the closed curves $\mathcal{O}_{0}, \mathcal{O}_{1}$.

The prime period function $p(\cdot)$ is continuous on the set $\left\{x \in \mathbb{T}^{2}\right.$ : $p(x)>0\}$. Using this fact we define a continuous function $h:\left[s_{0}, s_{1}\right] \times$ $[0,1] \rightarrow \mathbb{T}^{2}$ such that $h(s, t)=\varphi_{t p(\gamma(s))}(\gamma(s))$. Since $\cup_{0 \leq t \leq 1} h\left(s_{j}, t\right)=$ $\mathcal{O}_{j}, j=1,2$, we conclude that $h$ defines a continuous homotopy between $\mathcal{O}_{0}$ and $\mathcal{O}_{1}$. We claim that the image of $h$ does not intersect the interiors of $\mathcal{O}_{0}$ and $\mathcal{O}_{1}$. To prove the claim, suppose that $h\left(s^{\prime}, t^{\prime}\right)$ belongs, say, to the interior of $\mathcal{O}_{0}$. Then clearly $s^{\prime} \in\left(s_{0}, s_{1}\right)$. By our construction this means that $\gamma$ lies in the exterior of $\mathcal{O}_{0}$. So, $\gamma\left(s^{\prime}\right) \in$ exterior $\mathcal{O}_{0}$ and $\varphi_{t^{\prime} p\left(\gamma\left(s^{\prime}\right)\right)}\left(\gamma\left(s^{\prime}\right)\right) \in$ interior $\mathcal{O}_{0}$. This implies the existence of a point $t^{\prime \prime} \in[0,1]$ such that $\varphi_{t^{\prime \prime} p\left(\gamma\left(s^{\prime}\right)\right)}\left(\gamma\left(s^{\prime}\right)\right) \in \mathcal{O}_{0}$ and hence, $\gamma\left(s^{\prime}\right) \in \mathcal{O}_{0}$, a contradiction. This proves the claim.

To finish the proof of the proposition, attach another torus to $\mathbb{T}^{2}$ along the curve $\mathcal{O}_{0}$. We obtain a double-torus $\mathbb{T}^{2}+\mathbb{T}^{2}$. It follows from the claim above that $h$ is a homotopy of $\mathcal{O}_{0}$ into $\mathcal{O}_{1}$ on $\mathbb{T}^{2}+\mathbb{T}^{2}$. This is not possible, because the loops $\mathcal{O}_{0}$ and $\mathcal{O}_{1}$ belong to different classes 
of the fundamental group on $\mathbb{T}^{2}+\mathbb{T}^{2}$. This contradiction finishes the proof. Note that the same result with the identical proof holds on any domain in $\mathbb{R}^{2}$.

3. Proof of Lemma 10. We prove (25) by induction and take $t=k \in \mathbb{N}$ in (25). For $m=1$ this inequality (and even equality) is given in (4), see [CL, Thm. 8.15]. Assume that (25) holds for $m=1, \ldots, n-1$. For $k \in \mathbb{N}$ use the chain rule in [AMR, p. 97] for $\varphi \circ \varphi_{k-1}$ :

$$
\begin{aligned}
& D^{n}\left(\varphi \circ \varphi_{k-1}\right)(x)\left(v_{1}, \ldots, v_{n}\right) \\
& \quad=D^{n} \varphi\left(\varphi_{k-1} x\right)\left(D\left(\varphi_{k-1}\right)(x) v_{1}, \ldots, D\left(\varphi_{k-1}\right)(x) v_{n}\right)
\end{aligned}
$$

$$
\begin{gathered}
+\sum_{p=2}^{n-1} \sum_{j_{1}+\ldots+j_{p}=n} \sum_{\{\ell\}} D^{p} \varphi\left(\varphi_{k-1} x\right) \\
\left(D^{j_{1}}\left(\varphi_{k-1}\right)(x)\left(v_{\ell_{1}}, \ldots, v_{\ell_{j_{1}}}\right), \ldots, D^{j_{p}}\left(\varphi_{k-1}\right)(x)\left(v_{\ell_{j_{1}+\cdots+j_{p-1}+1}}, \ldots, v_{\ell_{p}}\right)\right) \\
+D \varphi\left(\varphi_{k-1} x\right) D^{n}\left(\varphi_{k-1}\right)(x)\left(v_{1}, \ldots, v_{n}\right) .
\end{gathered}
$$

The middle term in (27) does not contain derivatives of $\varphi$ of order $n$. By the induction assumption, we may apply estimate (26) for $m=$ $1, \ldots, n-1$. Since the number of summands in the middle term does not depend on $k$, we conclude that the norm of all terms in (27) except the last one is dominated by $c \exp [n(\Lambda+\epsilon) k]$. For the last term we again use the chain rule for $\varphi_{k-1}=\varphi \circ \varphi_{k-2}$ :

$$
\begin{aligned}
& D \varphi\left(\varphi_{k-1} x\right) D^{n}\left(\varphi \circ \varphi_{k-2}\right)(x)\left(v_{1}, \ldots, v_{n}\right) \\
& =D \varphi\left(\varphi_{k-1} x\right) D^{n} \varphi\left(\varphi_{k-2} x\right)\left(D\left(\varphi_{k-2}\right)(x) v_{1}, \ldots, D\left(\varphi_{k-2}\right)(x) v_{n}\right) \\
& +\sum_{p=2}^{n-1} \sum_{j_{1}+\cdots+j_{p}=n} \sum_{\{\ell\}} D \varphi\left(\varphi_{k-1} x\right) D^{p} \varphi\left(\varphi_{k-2} x\right) \\
& \quad\left(D^{j_{1}}\left(\varphi_{k-2}\right)(x)(\ldots), \ldots, D^{j_{p}}\left(\varphi_{k-2}\right)(x)(\ldots)\right) \\
& +D \varphi\left(\varphi_{k-1} x\right) D \varphi\left(\varphi_{k-2} x\right) D^{n}\left(\varphi_{k-2}\right)(x)\left(v_{1}, \ldots, v_{n}\right) .
\end{aligned}
$$

Again, by the induction assumption and (26), the norms of all terms, except the last one, are dominated by $c \exp [n(\Lambda+\epsilon) k]$. But the last term can be written as $D\left(\varphi_{2}\right)\left(\varphi_{k-2} x\right) D^{n}\left(\varphi_{k-2}\right)(x)(\ldots)$. We repeat this argument until the last term becomes $D\left(\varphi_{k-1}\right)(\varphi x) D^{n} \varphi(x)(\ldots)$. Since the total number of terms is growing not faster than polynomially in $k,(25)$ follows. 


\section{REFERENCES}

[AMR] R. Abraham, J. Marsden, and T. Ratiu, Manifolds, tensor analysis, and applications, Springer-Verlag, New York, 1988.

[C] S. Chandrasekhar, Hydrodynamic and hydromagnetic stability, Clarendon Press, Oxford, 1961.

[CL] C. Chicone and Y. Latushkin, Evolution Semigroups in Dynamical Systems and Differential Equations, Math. Surv. Monogr. 70, AMS, Providence, 1999.

[DH] P. G. Drazin and L. N. Howard, Hydrodynamic stability of parallel flow of an inviscid fluid, Adv. Appl. Math. 9, 1-89 (1966).

[DR] P. G. Drazin and W. H. Reid, Hydrodynamic stability. Cambridge, 1981.

[EE] D. E. Edmunds and W. D. Evans, Spectral Theory and Differential Operators, Clarendon Press, Oxford, 1989.

[EN] K. Engel and R. Nagel, One-parameter semigroups for linear evolution equations, Springer-Verlag, New York, 2000.

[FH] S. Friedlander and L. Howard, Instability in parallel flows revisited, Studies Appl. Math. 101, 1-21 (1998).

[FSV] S. Friedlander, W. Strauss, and M. Vishik, Nonlinear instability in an ideal fluid, Ann. Inst. H. Poincaré, Anal. non linéaire 14, 187-209 (1997).

[FSV2] S. Friedlander, W. Strauss, and M. Vishik, Robustness of instability for the two-dimensional Euler equations, SIAM J. Math. Anal. 30 (1999), 13431354 .

[FV] S. Friedlander and M. Vishik, Dynamo theory, vorticity generation, and exponential stretching, Chaos, 1 (1991), 198-205.

[FV2] S. Friedlander and M. Vishik, Instability criteria for steady flows of a perfect fluid, Chaos 2(3) (1992), 455-460.

[LH1] A. Lifschitz and E. Hameiri, Local stability conditions in fluid dynamics, Phys. Fluids A 3 (1991) 2644-2651.

[LH2] A. Lifschitz and E. Hameiri, Localized instabilities of vortex rings with swirl, Comm. Pure Appl. Math. 46 (1993) 1379-1408.

[LV] Y. Latushkin and M. Vishik, Linear stability in an ideal incompressible fluid, Comm. Math. Phys., 233 (2003), 439-461 (with M. Vishik).

[L] C. C. Lin, The theory of hydrodynamic stability, Cambridge, 1955.

$[\mathrm{N}] \quad$ R. Nussbaum, The radius of the essential spectrum, Duke Math. J. 37 (1970), 473-478.

[O] V. Oseledets, A multiplicative ergodic theorem. Lyapunov characteristic numbers for dynamical systems, Trans. Moscow Math. Soc. 19 (1968), 197231.

[RS] M. Reed and B. Simon, Methods of Modern Mathematical Physics. IV: Analysis of Operators, Academic Press, New York, 1978.

[S] R. Shvydkoy, On the essential spectrum of the linearized surface quasigeostrophic equation, in preparation.

[S2] R. Shvydkoy, A new proof of Vishik's formula, submitted to J. Math. Pures et Appl, 2003.

[SL] R. Shvydkoy and Y. Latushkin, The essential spectrum of the linearized 2D Euler operator is a vertical band, Contemp. Math., Proc. UAB 2002 Intl. Conf. Diff. Eqns. and Mathem. Phys., Y. Karpeshina, G. Stolz, R. Weikard, and Y. Zeng (edts), to appear. 
[V] M. M. Vishik, Spectrum of small oscillations of an ideal fluid and Lyapunov exponents, J. Math. Pures et Appl. 75 (1996), 531-558.

[VF] M. Vishik and S. Friedlander, Dynamo theory methods for hydrodynamic stability, J. Math. Pures Appl. 72 (1993), 145-180.

[Y] Y. Yudovich, The linearization method in hydrodynamical stability theory, Transl. Math. Monogr. 74, AMS, Providence, 1989.

Department of Mathematics, University of Missouri, Columbia, MO 65211

E-mail address: shvidkoy@math.missouri.edu, yuri@math.missouri.edu 